\title{
APEX CO(3-2) observations of NGC 6822
}

\author{
S. De Rijcke ${ }^{1, \star}$, P. Buyle ${ }^{1}$, J. Cannon ${ }^{2}$, F. Walter ${ }^{2}$, A. Lundgren ${ }^{3}$, D. Michielsen ${ }^{4}$, and H. Dejonghe ${ }^{1}$ \\ 1 Astronomical Observatory, Ghent University, Krijgslaan 289 - S9, 9000 Ghent, Belgium \\ e-mail: [Sven.DeRijcke;Pieter.Buyle]@uGent.be \\ 2 Max-Planck-Institut für Astronomie, Königstuhl 17, 69117 Heidelberg, Germany \\ 3 ESO, Casilla 19001, Santiago 19, Chile \\ ${ }^{4}$ University of Nottingham, University Park, Nottingham NG7 2RD, UK
}

Received 11 April 2006 / Accepted 1 June 2006

ABSTRACT

\begin{abstract}
We observed the ${ }^{12} \mathrm{CO}(3 \rightarrow 2)$ emission of the emission-line regions Hubble I, Hubble V, Hubble X, Holmberg 18, and the stellar emission-line object S28 in NGC 6822 with the ESO Atacama Pathfinder Experiment (APEX) $12 \mathrm{~m}$ telescope as part of its science verification. The very low system temperature of 130-180 K enabled us to achieve detections in 4 single pointings and in a high spatial resolution $70^{\prime \prime} \times 70^{\prime \prime}$ map of Hubble V. We compare the spectra with HI observations, obtained with the Australia Telescope Compact Array, of the same regions. In combination with previous multi-line $\mathrm{CO}$ observations, we perform a preliminary investigation of the physical conditions in Hubble V using a simple LTE model. We estimate the mass of the Hubble V region and the $\mathrm{H}_{2} / I_{\mathrm{CO}(3 \rightarrow 2)}$ conversion factor. Also, we show that Hubble $\mathrm{V}$ is located very near the line-width versus size relation traced by the Milky Way and LMC molecular clouds.
\end{abstract}

Key words. galaxies: individual: NGC 6822 - galaxies: ISM - telescopes

\section{Introduction}

We present ${ }^{12} \mathrm{CO}(3 \rightarrow 2)$ observations of the emission-line regions Hubble I, Hubble V, Hubble X, Holmberg 18, and the stellar emission-line object S28 (Killen \& Dufour 1982) in NGC 6822 with the ESO Atacama Pathfinder Experiment (APEX) $12 \mathrm{~m}$ telescope ${ }^{1}$. NGC 6822 is a Local Group dwarf irregular galaxy, of type IB(s)m. Its study started with the landmark paper of Hubble (1925), who identified it as a galaxy in its own right, external to the Milky Way. The most recent estimate of its distance, based on observations of 116 Cepheid variables, places it at $466 \pm 20 \mathrm{kpc}$ (Pietrzyński et al. 2004). Peimbert et al. (2005) find a metallicity $12+\log (\mathrm{O} / \mathrm{H})=8.37 \pm 0.09$ for Hubble $\mathrm{V}$ and $12+\log (\mathrm{O} / \mathrm{H})=8.19 \pm 0.16$ for Hubble $\mathrm{X}$, corresponding to roughly half the solar metallicity. Star formation proceeded at an almost constant rate up to the present, except for the central bar region, where star-formation increased by a factor of 3-4 during the last $600 \mathrm{Myr}$ (Wyder 2001). Its proximity allows us to study the different components and phases of its interstellar medium on scales of order 10-100 parsec.

The detection of the compact molecular clouds associated with Hubble V was first reported by Wilson (1994). Later on, the emission-line regions Hubble I, Hubble V, and Holmberg 18, and the stellar emission-line object S28 were observed in ${ }^{12} \mathrm{CO}(1 \rightarrow$ 0 ) emission by Israel (1997). Moreover, for Hubble V, the brightest HII region in NGC 6822, detections of ${ }^{12} \mathrm{CO}(2 \rightarrow 1)$,

\footnotetext{
* Postdoctoral Fellow of the Fund for Scientific Research - Flanders (Belgium)(F.W.O).

1 This publication is based on data acquired with the Atacama Pathfinder Experiment (APEX). APEX is a collaboration between the Max-Planck-Institut für Radioastronomie, the European Southern Observatory, and the Onsala Space Observatory.
}

${ }^{12} \mathrm{CO}(3 \rightarrow 2),{ }^{12} \mathrm{CO}(4 \rightarrow 3)$, and ${ }^{13} \mathrm{CO}(1 \rightarrow 0)$ have been reported (Israel et al. 2003). ${ }^{12} \mathrm{CO}(1 \rightarrow 0)$ and ${ }^{12} \mathrm{CO}(2 \rightarrow 1)$ observations centered on Hubble $X$, on the other hand, did not yield a detection (Israel et al. 2003). These results will serve as a comparison for the APEX data presented here.

In this letter, we combine the ${ }^{12} \mathrm{CO}(3 \rightarrow 2)$ line intensities measured with APEX with line intensities of other ${ }^{12} \mathrm{CO}$ and ${ }^{13} \mathrm{CO}$ transitions, taken from the literature, to constrain the physical conditions of the molecular interstellar medium of NGC 6822 using simple LTE models. We also investigate the spatial distribution of the ${ }^{12} \mathrm{CO}(3 \rightarrow 2)$ emission and how it correlates with previous high resolution $\mathrm{HI}$ observations.

\section{Observations and data reduction}

We observed the ${ }^{12} \mathrm{CO}(3 \rightarrow 2)$ line towards the star forming regions Hubble I, V, X, and Holmberg 18, and the stellar emission-line object S28 (Killen \& Dufour 1982) in NGC 6822 with the ESO Atacama Pathfinder Experiment (APEX) $12 \mathrm{~m}$ telescope on the nights of 17, 18, 20, 21, 25 and 26 August 2005 as part of its science verification. We used a Fast Fourier Transform Spectrometer (FFTS) backend built by MPIfR (Klein et al. 2006). The frequency was centered on $\mathrm{CO}(3 \rightarrow$ 2) $(345.79 \mathrm{GHz})$ and corrected for the systemic velocity of NGC 6822. The beamsize of the telescope at this frequency is 18.2" FWHM (Güsten et al. 2006). We used a $1 \mathrm{GHz}$ bandwidth divided over 8192 channels, corresponding to a velocity resolution of $0.1 \mathrm{~km} \mathrm{~s}^{-1}$ per channel. All targets were observed with a single pointing, except for Hubble $\mathrm{V}$, which was spatially mapped on a rectangular Nyquist $1^{\prime} \times 1^{\prime}$ grid. On-source integration times were $31.4 \mathrm{~min}$ (Hubble I), $9.1 \mathrm{~min}$ (single pointing of Hubble V), 1.0-10.4 min per pointing of the map of Hubble V, 
Table 1. $\mathrm{CO}(3 \rightarrow 2)$ properties of the targeted regions in NGC 6822: the peak main-beam temperature, $T_{\mathrm{mb}}$, the velocity of the line with respect to the Local Standard of Rest, the line FWHM, and the integrated line intensity, $I_{\mathrm{CO}}$.

\begin{tabular}{ccccccc}
\hline \hline Name & RA & Dec & $T_{\mathrm{mb}}(\mathrm{K})$ & LSR velocity $\left(\mathrm{km} \mathrm{s}^{-1}\right)$ & $F W H M\left(\mathrm{~km} \mathrm{~s}^{-1}\right)$ & $I_{\mathrm{CO}}\left(\mathrm{K} \mathrm{km} \mathrm{s}^{-1}\right)$ \\
\hline Hubble I & 194431.64 & -144201.2 & $0.07 \pm 0.02$ & $-66.3 \pm 0.4$ & $6.1 \pm 1.1$ & $0.49 \pm 0.11$ \\
Hubble V & 194452.80 & -144311.0 & $0.61 \pm 0.02$ & $-41.3 \pm 0.1$ & $6.0 \pm 0.2$ & $3.89 \pm 0.14$ \\
Hubble V & 194452.80 & -144311.0 & deconvolved & $-41.3 \pm 0.4$ & $6.0 \pm 0.2$ & $6.65 \pm 0.59$ \\
Hubble X & 194505.20 & -144313.0 & & & & $<0.30$ \\
Holmberg 18 & 194448.93 & -145238.0 & $0.11 \pm 0.02$ & $-43.6 \pm 0.2$ & $2.5 \pm 0.4$ & $0.29 \pm 0.07$ \\
KD82_S28 & 194457.79 & -144751.5 & $0.12 \pm 0.02$ & $-57.5 \pm 0.3$ & $5.2 \pm 0.7$ & $0.67 \pm 0.11$ \\
\hline
\end{tabular}

or $110.1 \mathrm{~min}$ in total, $3.1 \mathrm{~min}$ (Hubble $\mathrm{X}$ ), $21.9 \mathrm{~min}$ (Holmberg 18), and $22.3 \mathrm{~min}$ (S28). The rms of the pointing model was about $3^{\prime \prime}$. Pointing was regularly checked and updated on the nearby pointing source W-Aql. We used position switching. The reference position was set to $+480^{\prime \prime}$ in RA relative to the center position of each source. Dual side band (DSB) system temperatures were $128 \mathrm{~K}$ (Hubble I), $152 \mathrm{~K}$ (single pointing of Hubble V), 135-182 K (map of Hubble V), 133 K (Hubble X), 156 K (Holmberg 18), and 176 K (S28) (Risacher et al. 2006). The precipitable water vapour was $0.5-1.0 \mathrm{~mm}$, corresponding to a $\tau_{225}$ of $0.27-0.29$, during the observations. Calibration errors amount to $\sim 15 \%$. In order to enhance the signal-to-noise ratio without compromising the spectral resolution, the spectra were rebinned to a $0.8 \mathrm{~km} \mathrm{~s}^{-1}$ resolution and they have been rectified over the velocity range $[-100,0] \mathrm{km} \mathrm{s}^{-1}$.

The data reduction was performed with the standard data analysis program GILDAS of the $30 \mathrm{~m}$ IRAM radio telescope. Observations related to the same pointing were first added together. Afterwards a polynomial of 4th order was fitted to an emission line free region of the spectral baseline and subtracted off this baseline. Some spectra still showed a residual double sinusoidal variation. One of these ripples arose due to a vibration of the gore-tex membrane that covers the entrance window to the Cassegrain cabin (L. Nyman, priv. comm.) and the other due to the vibration of the cold head of the closed-cycle cooling machine, which affected the LO coupling of the receiver, causing the receiver gain to vary (Risacher et al. 2006). We fitted a combination of two sine functions to those spectral baselines that contained this sinusoidal variation, omitting the spectral region around the $\mathrm{CO}(3 \rightarrow 2)$ emission line, and subtracted this off the spectrum. The antenna temperatures, $T_{\mathrm{A}}^{*}$, were converted to to main-beam brightness temperatures $\left(T_{\mathrm{mb}}=T_{\mathrm{A}}^{*} / \eta_{\mathrm{mb}}\right)$, using the main-beam efficiency $\eta_{\mathrm{mb}}=0.7$.

\section{Discussion}

We fitted Gaussians to the detected emission lines in order to estimate the peak intensity, $T_{\mathrm{mb}}(\mathrm{K})$, and the integrated intensity, $I_{\mathrm{CO}}=\int T_{\mathrm{mb}}(v) \mathrm{d} v\left(\mathrm{~K} \mathrm{~km} \mathrm{~s}^{-1}\right)$, of the ${ }^{12} \mathrm{CO}(3 \rightarrow 2)$ emission lines of the observed star-forming regions (see Table 1). We used the best fitting Gaussian and the $1 \sigma$ noise on the spectrum, estimated from the spectral region between -100 and $0 \mathrm{~km} \mathrm{~s}^{-1}$ and excluding the emission line, to generate 1000 new noisy spectra. These were analysed the same way as the original spectrum, allowing us to estimate the $1 \sigma$ errors on these quantities. For the non-detected star-forming region Hubble $\mathrm{X}$, we give a $3 \sigma$ upper limit over a velocity width of $6 \mathrm{~km} \mathrm{~s}^{-1}$.

\subsection{Physical conditions in Hubble $V$}

We made a preliminary assessment of the physical conditions in the $\mathrm{CO}$ cloud associated with Hubble $\mathrm{V}$ assuming local thermodynamical equilibrium (LTE). In that case, there is one excitation temperature, $T_{\text {ex }}$ responsible for populating the energy levels of the ${ }^{12} \mathrm{CO}$ and ${ }^{13} \mathrm{CO}$ isotopomers. This need of course not be the case in reality, with the higher- $J$ lines not being thermalized due to their larger Einstein $A$ coefficients. In the following, we will assume the isotopic ratio $X={ }^{12} \mathrm{CO} /{ }^{13} \mathrm{CO}=60$ (Langer \& Penzias 1993; Savage et al. 2002), in which case the optical depths of ${ }^{12} \mathrm{CO}$, denoted by $\tau_{12}$, and ${ }^{13} \mathrm{CO}$, denoted by $\tau_{13}$, obey the relation $\tau_{12}=X \tau_{13}$. The calculated line intensities are coupled to the observed quantities by the beam filling factor $f_{\mathrm{b}}$, which we assume to be the same for the ${ }^{12} \mathrm{CO}$ and ${ }^{13} \mathrm{CO}$ emission. Furthermore, we will assume that both the ${ }^{12} \mathrm{CO}$ and the ${ }^{13} \mathrm{CO}$ emission arises in the same region so that $\Omega_{\text {source }}$, the solid angle spanned on the sky by the CO source, is the same for both isotopomers. This is to keep this preliminary modeling as simple as possible since there is no physical reason why $f_{\mathrm{b}}$ and $\Omega_{\text {source }}$ should be the same for all transitions. We can use this source solid angle to correct the observed emission line brightness temperatures for beam dilution using the relation $T_{\mathrm{mb}}^{\prime}=T_{\mathrm{mb}}\left(\Omega_{\text {source }}+\Omega_{\text {beam }}\right) / \Omega_{\text {source }}$, with $\Omega_{\text {beam }}$ the beam solid angle. The main-beam brightness temperature of an observed transition can be written as

$$
T_{\mathrm{mb}, i}^{\prime}=f_{\mathrm{b}}\left(1-\mathrm{e}^{-\tau_{i}}\right) \frac{h v_{i}}{k}\left(\frac{1}{\mathrm{e}^{h v_{i} / k T_{\mathrm{ex}}}-1}-\frac{1}{\mathrm{e}^{h v_{i} / k T_{\mathrm{cmb}}}-1}\right),
$$

with $v_{i}$ the frequency of the transition, $\tau_{i}$ its optical depth, and $T_{\text {cmb }}=2.725 \mathrm{~K}$ the background radiation temperature.

Using a non-linear minimisation routine, we simultaneously fitted a Gaussian model for the spatial distribution of the CO emission of Hubble V, convolved with the APEX beam, which constrains the source solid angle $\Omega_{\text {source }}$ and the parameters $T_{\mathrm{ex}}$, $f_{\mathrm{b}}$, and $\tau_{12}$ to the ${ }^{12} \mathrm{CO}(3 \rightarrow 2)$ map, presented in Fig. 2 , and to the ${ }^{12} \mathrm{CO}(3 \rightarrow 2)$ brightness temperature measured by us, and the ${ }^{12} \mathrm{CO}(1 \rightarrow 0),{ }^{12} \mathrm{CO}(2 \rightarrow 1),{ }^{12} \mathrm{CO}(4 \rightarrow 3)$, and ${ }^{13} \mathrm{CO}(1 \rightarrow 0)$ brightness temperatures presented in Israel et al. (2003). We then used the best fitting values for $\Omega_{\text {source }}, T_{\mathrm{ex}}, f_{\mathrm{b}}$, and $\tau_{12}$ to generate 10000 mock data sets with added Gaussian noise on each of the observed quantities, using the measured $1 \sigma$ uncertainties on the measured quantities as estimates for the dispersions of each of the noise distributions. These mock data sets were analysed the same way as the original set, allowing us to estimate the $1 \sigma$ errors on the derived quantities. This way, we find that the parameter values $\Omega_{\text {source }}=209 \pm 50 \mathrm{arcsec}^{2}$, $T_{\text {ex }}=49 \pm 27 \mathrm{~K}, \tau_{12}=3.7 \pm 2.3$, and $f_{\mathrm{b}}=0.04 \pm 0.03$ provide the best fit to the whole data-set. There is a large degree of degeneracy between the parameters of this model, e.g. between $T_{\mathrm{ex}}$ and $f_{\mathrm{b}}$. This is reflected by the very large errorbars on these quantities. Still, the minimisation routine converges to the same solution independent of the starting point of the minimisation which proves that the minimum of the $\chi^{2}$ is well defined. Moreover, this temperature estimate agrees with the dust temperature derived from the ratio of the $60 \mu$ and $100 \mu$ IRAS flux 

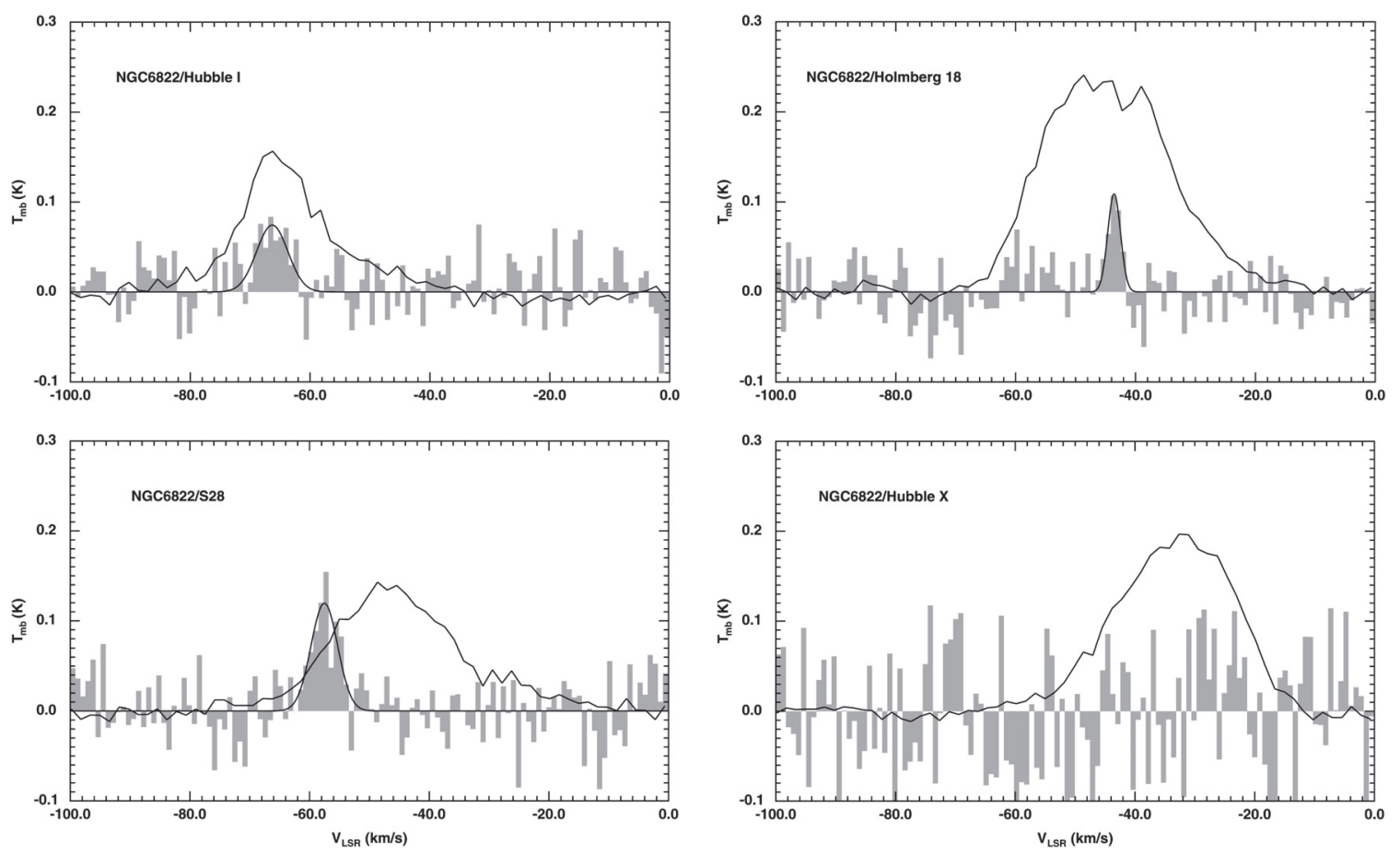

Fig. 1. $\mathrm{CO}(3 \rightarrow 2)$ spectra of the star-forming regions Hubble I, Hubble X, and Holmberg 18 and the stellar emission-line object S28 in NGC 6822, overplotted with the best fitting Gaussian. The black curve indicates the HI emission as derived by de Blok \& Walter (2006). Evidently, the molecular gas associated with Hubble I and Holmberg 18 has the same velocity as the neutral gas. The velocity of the molecular gas associated with the stellar object S28 differs from that of the HI. Hubble X was not detected.

densities, $f_{v}(60)=7.89 \mathrm{Jy}$ and $f_{v}(100)=11.81 \mathrm{Jy}$, of Hubble $\mathrm{V}$, $T_{\text {dust }} \approx 40 \mathrm{~K}$. This estimate was derived assuming a single temperature component and a $\lambda^{-1}$ emissivity law. Given the apparently rather high temperature of this $\mathrm{CO}$ emission cloud, observations of higher- $J$ transitions, e.g. with FLASH or the future SIS heterodyne receivers, are required for a more precise assessment of its physical properties using more sophisticated LVG models, taking into account non-LTE effects. Also, some of the published high- $J$ transition temperatures, such as ${ }^{12} \mathrm{CO}(4 \rightarrow 3)$ value of Israel et al. (2003), may be affected by the small area that was mapped. If some of the emission was missed, this may lead to an underestimation of the brightness temperature of these transitions.

Using the ${ }^{12} \mathrm{CO}(3 \rightarrow 2) F W H M$ line-width, $\Delta V$, in $\mathrm{km} \mathrm{s}^{-1}$, and the radius of the emission region, $R$, in parsec, we can also estimate the virial mass of Hubble $\mathrm{V}$ as $M_{\mathrm{vir}} \approx 190 R(\Delta V)^{2}$, in solar masses (MacLaren et al. 1988). For $\Delta V=6.0 \mathrm{~km} \mathrm{~s}^{-1}, R=$ $\sqrt{\Omega_{\text {source }}} / 2.355=6.1^{\prime \prime}=13.9 \mathrm{pc}$, we find $M_{\text {vir }} \approx 9.5 \times 10^{4} M_{\odot}$. Using the relation $M_{\text {dust }}=1.27 f_{v}(100) D^{2}(\exp (144 K / T)-1) M_{\odot}$ (Boselli et al. 2002) for the dust mass, with $D$ the distance in $\mathrm{Mpc}$, we find $M_{\text {dust }} \approx 130 M_{\odot}$. Using the metallicitydependent gas versus dust mass relation (Eq. (6) in Boselli et al. 2002), this yields $M_{\text {gas }} \approx 9 \times 10^{4} M_{\odot}$, in good agreement with the virial mass. This is much less than the estimate of the total gas mass $M_{\text {gas }}=10 \pm 5 \times 10^{5} M_{\odot}$ of Israel et al. (2003). This is most likely because we are measuring quantities (radius, velocity dispersion) that pertain only to the $\mathrm{CO}$ emission region: any mass distribution outside this region, which was taken into account by Israel et al. (2003), would not have a very large effect on the virial mass estimate. Hubble $\mathrm{V}$ is also very near the line-width versus size relation traced by the Milky Way and LMC molecular clouds (Heikkila 1995; Maloney 1990). We estimate the ${ }^{12} \mathrm{CO}(3 \rightarrow 2)$ to $\mathrm{H}_{2}$ conversion at $5.4-5.8 \times 10^{20} \mathrm{~cm}^{-2}$

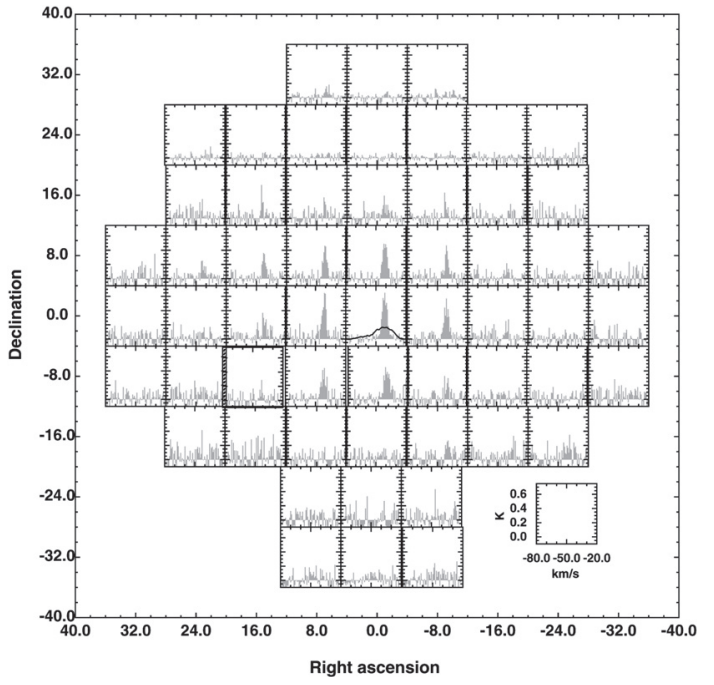

Fig. 2. ${ }^{12} \mathrm{CO}(3 \rightarrow 2)$ map of Hubble V. Each panel shows the brightness temperature $T_{\mathrm{mb}}$ as a function of velocity with respect to the Local Standard of Rest (LSR). Some spectra still show some residual variations even after subtracting off a double sinusoidal baseline. Nearest neighbor panels overlap by $8^{\prime \prime}$, i.e. about half of the APEX beam width at this frequency, so that next nearest neighbor panels are roughly independent. The CO source associated with Hubble V is clearly resolved in this map and is extended towards the north-east.

$\left(\mathrm{K} \mathrm{km} \mathrm{s}^{-1}\right)^{-1}$, depending on whether the deconvolved or the observed brightness is used.

\subsection{Comparison with previous observations}

In Figs. 1 and 2, we plot the (unscaled) HI spectra derived by de Blok \& Walter (2006), of the same regions 
Table 2. Comparison of the LTE model with the measured intensities of the ${ }^{12} \mathrm{CO}$ and ${ }^{13} \mathrm{CO}$ transitions. All intensities have been corrected for beam dilution using $\Omega_{\text {source }}=209 \operatorname{arcsec}^{2}$.

\begin{tabular}{ccc}
\hline \hline Transition & Measurement $(\mathrm{K})$ & Model $(\mathrm{K})$ \\
\hline${ }^{12} \mathrm{CO}(1 \rightarrow 0)$ & $1.72 \pm 0.11$ & 1.73 \\
${ }^{12} \mathrm{CO}(2 \rightarrow 1)$ & $1.69 \pm 0.08$ & 1.66 \\
${ }^{12} \mathrm{CO}(3 \rightarrow 2)$ & $1.56 \pm 0.05$ & 1.57 \\
${ }^{12} \mathrm{CO}(4 \rightarrow 3)$ & $1.50 \pm 0.09$ & 1.48 \\
${ }^{13} \mathrm{CO}(1 \rightarrow 0)$ & $0.11 \pm 0.07$ & 0.11 \\
\hline
\end{tabular}

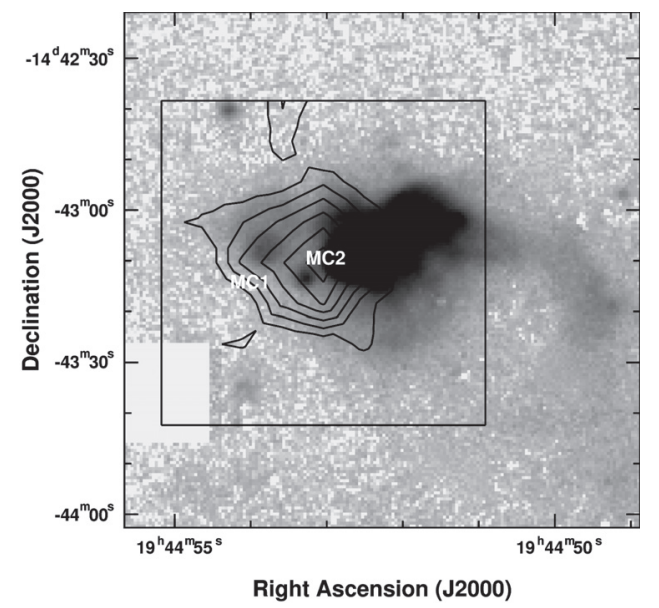

Fig. 3. Spatial distribution of the $\mathrm{CO}(3 \rightarrow 2)$ emission of Hubble $\mathrm{V}$ at $18^{\prime \prime}$ resolution (contours) plotted over an $\mathrm{H} \alpha$ image (greyscale). The square indicates the region mapped by our APEX observations. The contour values are 1 (which corresponds to $3 \sigma$ ), 1.5, 2, 2.5, 3.0, and $3.5 \mathrm{~K} \mathrm{~km} \mathrm{~s}^{-1}$. The $\mathrm{CO}$ emission is not centered on the $\mathrm{H} \alpha$ emission. The positions of the $\mathrm{CO}$ clouds $\mathrm{MC} 1$ and $\mathrm{MC} 2$, identified by Wilson (1994), are indicated in the figure.

as our observations on top of the ${ }^{12} \mathrm{CO}(3 \rightarrow 2)$ spectra. One can see that apart from S28, both emission lines are coincidental although the $\mathrm{HI}$ emission is systematically broader than the ${ }^{12} \mathrm{CO}(3 \rightarrow 2)$ emission. In Fig. 3, we plot the spatial distribution of the ${ }^{12} \mathrm{CO}(3 \rightarrow 2)$ emission of Hubble $\mathrm{V}$ at $18^{\prime \prime}$ resolution. We find a different morphology than the one derived by Israel et al. (2003), however it is in accordance with their [CII] emission, found in the same paper. A possible cause might be the higher system temperature of $2460 \mathrm{~K}$ during their observation, producing more noise which might cause a shift in the spatial distribution. The main emission peak in our map corresponds to the molecular cloud MC2, first detected by Wilson (1994); the eastward extension corresponds only very roughly to MC1.

With our observations we prove that APEX is very suited for deriving spatially extended, high signal-to-noise maps of emission-line regions in Local Group dwarf galaxies, where one can achieve a spatial resolution of a few tens of parsecs.

Acknowledgements. We thank Erwin de Blok for kindly permitting us to use the $\mathrm{H} \alpha$ image of Hubble V and the radio spectra of the different NGC 6822 fields. We thank the referee, Jonathan Braine, for his valuable remarks.

\section{References}

Boselli, A., Lequeux, J., \& Gavazzi, G. 2002, A\&A, 384, 33 de Blok, W. J. G., \& Walter, F. 2006, AJ, 131, 343

Güsten, R., Nyman, L. Å., Schilke, P., et al. 2006, A\&A, 454, L13

Heikkilä, A. 1995, The XXVIIth Young European Radio Astronomers Conference, ed. D. A. Green, \& W. Steffen (Cambridge: University Press) Hubble, E. 1925, ApJ, 62, 409

Israel, F. P. 1997, A\&A, 317, 65

Israel, F. P., Baas, F., Rudy, R. J., Skillman, E. D., \& Woodward, C. E. 2003, A\&A, 397, 97

Killen, R. M., \& Dufour, R. J. 1982, PASP, 94, 444

Klein, B., Philipp, S. D., Kraemer, I., et al. 2006, A\&A, 454, L29

Langer, W. D., \& Penzias, A. A. 1993, ApJ, 408, 539

MacLaren, I., Richardson, K. M., \& Wolfendale, A. W. 1988, ApJ, 333, 821

Maloney, P. 1990, ApJ, 348, L9

Peimbert, A., Peimbert, M., \& Ruiz, M. T. 2005, ApJ, 634, 1056

Pietrzyński, G., Gieren, W., Udalski, A., et al. 2004, AJ, 128, 2815

Risacher, C., Vassilev, V., Monje, R., et al. 2006, A\&A, 454, L17

Savage, C., Apponi, A. J., \& Ziurys, L. M. 2002, ApJ, 578, 211

Wilson, C. D. 1994, ApJ, 434, L11

Wyder, T. K. 2001, AJ, 122, 2490 